\title{
Convergence of the parabolic complex Monge-Ampère equation on compact Hermitian manifolds
}

\author{
Matt GiLL
}

\begin{abstract}
We prove $C^{\infty}$ convergence for suitably normalized solutions of the parabolic complex Monge-Ampère equation on compact Hermitian manifolds. This provides a parabolic proof of a recent result of Tosatti and Weinkove.
\end{abstract}

\section{Introduction}

Let $(M, g)$ be a compact Hermitian manifold of complex dimension $n$ and $\omega$ be the real $(1,1)$ form $\omega=\sqrt{-1} \sum_{i, j} g_{i \bar{j}} d z^{i} \wedge d z^{\bar{j}}$. Let $F$ be a smooth function on $M$. We consider the parabolic complex Monge-Ampère equation

$$
\frac{\partial \varphi}{\partial t}=\log \frac{\operatorname{det}\left(g_{i \bar{j}}+\partial_{i} \partial_{\bar{j}} \varphi\right)}{\operatorname{det} g_{i \bar{j}}}-F, \quad g_{i \bar{j}}+\partial_{i} \partial_{\bar{j}} \varphi>0,
$$

with initial condition $\varphi(x, 0)=0$.

The study of this type of Monge-Ampère equation originated in proving the Calabi conjecture. The proof of the conjecture reduced to assuming that $\omega$ is Kähler and finding a unique solution to the elliptic Monge-Ampère equation

$$
\log \frac{\operatorname{det}\left(g_{i \bar{j}}+\partial_{i} \partial_{\bar{j}} \varphi\right)}{\operatorname{det} g_{i \bar{j}}}=F, \quad g_{i \bar{j}}+\partial_{i} \partial_{\bar{j}} \varphi>0 .
$$

Calabi showed that if a solution to (1.2) exists, it is unique up to adding a constant to $\varphi$ [3]. Yau used the continuity method to show that if

$$
\int_{M} \mathrm{e}^{F} \omega^{n}=\int_{M} \omega^{n}
$$

then (1.2) admits a smooth solution [29]. The proof of Yau required a priori $C^{\infty}$ estimates for $\varphi$. 
Cao used Yau's estimates to show that in the Kähler case, (1.1) has a smooth solution for all time that converges to the unique solution of (1.2) [4].

Since not every complex manifold admits a Kähler metric, one can naturally study the Monge-Ampère Equations (1.1) and (1.2) on a general Hermitian manifold. Fu and Yau discussed physical motivation for studying non-Kähler metrics in a recent paper [11].

Cherrier studied (1.2) in the general Hermitian setting in the eighties, and showed that in complex dimension 2 or when $\omega$ is balanced (i.e., $\left.d\left(\omega^{n-1}\right)=0\right)$, there exists a unique normalization of $F$ such that (1.2) has a unique solution [7]. Precisely, Cherrier proved that under the above conditions, given a smooth function $F$ on $M$, there exists a unique real number $b$ and a unique function $\varphi$ solving the Monge-Ampère equation

$$
\log \frac{\operatorname{det}\left(g_{i \bar{j}}+\partial_{i} \partial_{\bar{j}} \varphi\right)}{\operatorname{det} g_{i \bar{j}}}=F+b, \quad g_{i \bar{j}}+\partial_{i} \partial_{\bar{j}} \varphi>0
$$

such that $\int_{M} \varphi \omega^{n}=0$.

Recently, Guan and Li proved that (1.2) has a solution on a Hermitian manifold with the added condition

$$
\partial \bar{\partial} \omega^{k}=0
$$

for $k=1,2$. They applied this result to finding geodesics in the space of Hermitian metrics. Related work can be found in $[2,5,6,9,14,15,19-21]$.

Tosatti and Weinkove gave an alternate proof of Cherrier's result in [25]. In a very recent paper [26], they showed that the balanced condition is not necessary and the result holds on a general Hermitian manifold. Dinew and Kolodziej studied (1.2) in the Hermitian setting with weaker conditions on the regularity of $F[8]$.

In this paper, we prove the following theorem.

Theorem 1.1. Let $(M, g)$ be a compact Hermitian manifold of complex dimension $n$ with $\operatorname{Vol}(M)=\int \omega^{n}=1$. Let $F$ be a smooth function on $M$. There exists a smooth solution $\varphi$ to the parabolic complex Monge-Ampère Equation (1.1) for all time. Let

$$
\tilde{\varphi}=\varphi-\int_{M} \varphi \omega^{n}
$$

Then $\tilde{\varphi}$ converges in $C^{\infty}$ to a smooth function $\tilde{\varphi}_{\infty}$. Moreover, there exists a unique real number $b$ such that the pair $\left(b, \tilde{\varphi}_{\infty}\right)$ is the unique solution to $(1.3)$. 
We remark that the main theorem gives a parabolic proof of the result due to Tosatti and Weinkove in [26].

The flow (1.1) could be considered as an analogue to Kähler-Ricci flow for Hermitian manifolds. In the special case that $-\sqrt{-1} \partial \bar{\partial} \log \operatorname{det} g=$ $\sqrt{-1} \partial \bar{\partial} F$ (such an $F$ always exists under the topological condition $c_{1}^{B C}$ $(M)=0$, e.g.) then taking $\sqrt{-1} \partial \bar{\partial}$ of the flow (1.1) yields

$$
\frac{\partial \omega^{\prime}}{\partial t}=\sqrt{-1} \partial \bar{\partial} \log \operatorname{det} g^{\prime}
$$

with initial condition $\omega^{\prime}(0)=\omega$. In general, the right-hand side is the first Chern form, but if we assume Kähler, it becomes $-\operatorname{Ric}\left(\omega^{\prime}\right)$.

When $(M, g)$ is Kähler, Székelyhidi and Tosatti showed that a weak plurisubharmonic solution to (1.2) is smooth using the parabolic flow (1.1) [24]. Their result suggests that the flow could be used to prove a similar result in the Hermitian case. In a recent paper [23], Streets and Tian consider a different parabolic flow on Hermitian manifolds and suggest geometric applications for the flow.

We now give an outline of the proof of the main theorem and discuss how it differs from previous results. In Sections 2 through 5, we build up theorems that eventually show that $\varphi$ is smooth. Like in Yau's proof, we derive lower-order estimates and then apply Schauder estimates to attain higher regularity for the solution.

In Section 2, we use the maximum principle to show that the time derivative of $\varphi$ is uniformly bounded. We define the normalization

$$
\tilde{\varphi}=\varphi-\int_{M} \varphi \omega^{n} .
$$

We chose to assume that the volume of $M$ is one to simplify the notation of this normalization and the following calculations. Then using the zerothorder estimate from [26], we prove that $\tilde{\varphi}$ is uniformly bounded.

Section 3 contains a proof of the second-order estimate. Specifically, we derive that

$$
\begin{aligned}
& \operatorname{tr}_{g} g^{\prime} \leq C_{1} \mathrm{e}^{C_{2}\left(\sup _{M \times[0, T)} \tilde{\varphi}-\inf _{M \times[0, T)} \tilde{\varphi}\right)} \\
& \times \mathrm{e}^{\left(\mathrm{e}^{A\left(\sup _{M \times[0, T)} \tilde{\varphi}-\inf _{M \times[0, T)} \tilde{\varphi}\right)}-\mathrm{e}^{A\left(\sup _{M \times[0, T)} \tilde{\varphi}-\tilde{\varphi}\right)}\right)},
\end{aligned}
$$

where $[0, T)$ is the maximum interval of existence for $\varphi$ and $C_{1}, C_{2}$ and $A$ are uniform constants. This estimate is not as sharp as the estimate

$$
\operatorname{tr}_{g} g^{\prime} \leq C \mathrm{e}^{\left(\mathrm{e}^{A\left(\sup _{M} \varphi-\inf _{M} \varphi\right)}-\mathrm{e}^{A\left(\sup _{M} \varphi-\varphi\right)}\right)},
$$


from Guan and Li or the estimate

$$
\operatorname{tr}_{g} g^{\prime} \leq C \mathrm{e}^{A\left(\varphi-\inf _{M} \varphi\right)}
$$

from Tosatti and Weinkove in the case $n=2$ or $\omega$ balanced. Cherrier also produced a different estimate. These estimates are from the elliptic case, but they suggest that (1.5) could be improved. The proof of (1.5) follows along the method of Tosatti and Weinkove in [25], but there are extra terms to control that arrive in the parabolic case.

In Section 5, we derive a Hölder estimate for the time-dependent metric $g_{i \bar{j}}^{\prime}$. This estimate provides higher regularity using a method of Evans [10] and Krylov [16]. To prove the Hölder estimate, we apply a theorem of Lieberman [17], a parabolic analogue of an inequality from Trudinger [27]. The method follows closely with the proof of the analogous estimate in [25], but differs in controlling the extra terms that arise from the time dependence of $\varphi$.

We show that $\varphi$ is smooth and also prove the long-time existence of the flow (1.1) in Section 5. The proof uses a standard bootstrapping argument.

Section 6 uses analogues of lemmas from $\mathrm{Li}$ and Yau [18] to prove a Harnack inequality for the equation

$$
\frac{\partial u}{\partial t}=g^{\prime i \bar{j}} \partial_{i} \partial_{j} u
$$

where $g^{\prime i \bar{j}} \partial_{i} \partial_{\bar{j}}$ is the complex Laplacian with respect to $g^{\prime}$. This differs from the equation

$$
\left(\triangle-q(x, t)-\frac{\partial}{\partial t}\right) u(x, t)=0
$$

considered by $\mathrm{Li}$ and Yau, where $\triangle$ is the Laplace-Beltrami operator.

In Section 7, we apply these lemmas to show that time derivative of $\tilde{\varphi}$ decays exponentially. Precisely, we show that

$$
\left|\frac{\partial \tilde{\varphi}}{\partial t}\right| \leq C e^{-\eta t}
$$

for some $\eta>0$. From here we show that $\tilde{\varphi}$ converges to a smooth function $\tilde{\varphi}_{\infty}$ as $t$ tends to infinity. In fact, the convergence occurs in $C^{\infty}$ and $\tilde{\varphi}_{\infty}$ is part of the unique pair $\left(b, \tilde{\varphi}_{\infty}\right)$ solving the elliptic Monge-Ampère equation

$$
\log \frac{\operatorname{det}\left(g_{i \bar{j}}+\partial_{i} \partial_{\bar{j}} \tilde{\varphi}_{\infty}\right)}{\operatorname{det} g_{i \bar{j}}}=F+b,
$$


where

$$
b=\int_{M}\left(\log \frac{\operatorname{det}\left(g_{i \bar{j}}+\partial_{i} \partial_{\bar{j}} \tilde{\varphi}_{\infty}\right)}{\operatorname{det} g_{i \bar{j}}}-F\right) \omega^{n} .
$$

This provides an alternate proof of the main theorem in [26].

\section{Preliminary estimates}

By standard parabolic theory, there exists a unique smooth solution $\varphi$ to (1.1) on a maximal-time interval $[0, T)$, where $0<T \leq \infty$.

We show that the time derivatives of $\varphi$ and its normalization $\tilde{\varphi}$ are bounded. This fact will be used in the second-order estimate.

Theorem 2.1. For $\varphi$ a solution of (1.1) and $\tilde{\varphi}$ as in (1.4),

$$
\left|\frac{\partial \varphi}{\partial t}\right| \leq C, \quad\left|\frac{\partial \tilde{\varphi}}{\partial t}\right| \leq C,
$$

where $C$ depends only on the initial data.

Proof. Differentiating (1.1) with respect to $t$ gives

$$
\frac{\partial \varphi_{t}}{\partial t}=g^{\prime i \bar{j}} \partial_{i} \partial_{\bar{j}} \varphi_{t}
$$

where $\varphi_{t}=\frac{\partial \varphi}{\partial t}$. So by the maximum principle,

$$
\left|\frac{\partial \varphi}{\partial t}(x, t)\right| \leq C \sup _{x \in M}\left|\frac{\partial \varphi}{\partial t}(x, 0)\right| .
$$

From the definition of $\tilde{\varphi}$,

$$
\left|\frac{\partial \tilde{\varphi}}{\partial t}\right| \leq\left|\frac{\partial \varphi}{\partial t}\right|+\int\left|\frac{\partial \varphi}{\partial t}\right| \omega^{n} \leq 2 C .
$$

We show that $\tilde{\varphi}$ is bounded in $M \times[0, T)$ using the main theorem of [26].

Theorem 2.2. For $\varphi$ a solution to (1.1) and $\tilde{\varphi}$ the normalized solution, there exists a uniform constant $C$ such that

$$
\sup _{M \times[0, T)}|\tilde{\varphi}| \leq C,
$$

where $[0, T)$ is the maximum interval of existence for $\varphi$. 
Proof. We can rearrange (1.1) to

$$
\log \frac{\operatorname{det} g_{i \bar{j}}^{\prime}}{\operatorname{det} g_{i \bar{j}}}=F-\frac{\partial \varphi}{\partial t}
$$

Since $\left|\frac{\partial \varphi}{\partial t}\right|$ is bounded by the previous theorem, this is equivalent to the complex Monge-Ampère equation of the main theorem in [26]. This implies that $\sup _{M} \varphi(., t)-\inf _{M} \varphi(., t) \leq C$ for some $C$ depending only on $(M, g)$ and $F$.

Fix $(x, t)$ in $M \times[0, T)$. Since $\int_{M} \tilde{\varphi} \omega^{n}=0$, there exists $(y, t)$ such that $\tilde{\varphi}(y, t)=0$. Then

$$
|\tilde{\varphi}(x, t)|=|\tilde{\varphi}(x, t)-\tilde{\varphi}(y, t)|=|\varphi(x, t)-\varphi(y, t)| \leq C .
$$

Thus $\tilde{\varphi}$ is a bounded function on $M \times[0, T)$.

\section{The second-order estimate}

In this section, $\triangle=g^{i \bar{j}} \partial_{i} \partial_{\bar{j}}$ will denote the complex Laplacian corresponding to $g$. Similarly, write $\triangle^{\prime}=g^{\prime i \bar{j}} \partial_{i} \partial_{j}$ for the complex Laplacian for the time dependent metric $g^{\prime}$. We prove an estimate on $\operatorname{tr}_{g} g^{\prime}=g^{i \bar{j}} g_{i \bar{j}}^{\prime}=n+\triangle \tilde{\varphi}$.

Theorem 3.1. For $\varphi$ a solution to (1.1) and $\tilde{\varphi}$ the normalized solution, we have the following estimate

$$
\begin{aligned}
\operatorname{tr}_{g} g^{\prime} \leq & C_{1} e^{C_{2}\left(\sup _{M \times[0, T)} \tilde{\varphi}-\inf _{M \times[0, T)} \tilde{\varphi}\right)} \\
& \times e^{\left(\mathrm{e}^{A\left(\sup _{M \times[0, T)} \tilde{\varphi}-\inf _{M \times[0, T)} \tilde{\varphi}\right)}-\mathrm{e}^{A\left(\sup _{M \times[0, T)} \tilde{\varphi}-\tilde{\varphi}\right)}\right)},
\end{aligned}
$$

where $[0, T)$ is the maximum interval of existence for $\varphi$ and $C_{1}, C_{2}$, and $A$ are uniform constants. Hence, there exists a uniform constant $C$ such that $\operatorname{tr}_{g} g^{\prime} \leq C$ and also

$$
\frac{1}{C} g \leq g^{\prime} \leq C g
$$

Proof. This proof follows along with the notation and method featured in [25]. For brevity we omit some of the calculations and refer the reader to 
$[12,25]$. Let $E_{1}$ and $E_{2}$ denote error terms of the form

$$
\begin{aligned}
& \left|E_{1}\right| \leq C_{1} \operatorname{tr}_{g^{\prime}} g, \\
& \left|E_{2}\right| \leq C_{2}\left(\operatorname{tr}_{g^{\prime}} g\right)\left(\operatorname{tr}_{g} g^{\prime}\right),
\end{aligned}
$$

where $C_{1}$ and $C_{2}$ are constants depending only on the initial data. We call such a constant depending only on $(M, g)$ and $\sup _{M} F$ a uniform constant. We remark that by the flow Equation (1.1) and estimate (2.1), an error term of type $E_{1}$ is also of type $E_{2}$ and a uniform constant is of type $E_{1}$. In general, $C$ will denote a uniform constant whose definition may change from line to line. For a function $\varphi$ on $M$, we write $\varphi_{i}$ for the ordinary derivative

$$
\varphi_{i}=\partial_{i} \varphi
$$

Similarly, $\varphi_{t}$ will denote the time derivative of $\varphi$. If $f$ is a function on $M$, we write $\partial f$ for the vector of ordinary derivatives of $f$.

We define the quantity

$$
Q=\log \operatorname{tr}_{g} g^{\prime}+\mathrm{e}^{A\left(\sup _{M \times[0, T)} \tilde{\varphi}-\tilde{\varphi}\right)} .
$$

We note that the form of $Q$ differs here than in [25], Yau's estimate [29] and Aubin's estimate [1]. They consider a quantity of the form $\log \operatorname{tr}_{g} g^{\prime}-A \varphi$. The exponential in the definition of $Q$ helps to control a difficult term in the analysis.

Fix $t^{\prime} \in[0, T)$. Then let $\left(x_{0}, t_{0}\right)$ be the point in $M \times\left[0, t^{\prime}\right]$ where $Q$ attains its maximum. Notice that if $t_{0}=0$ the result is immediate, so we assume $t_{0}>0$. To start the proof, we need to perform a change of coordinates made possible by the following lemma from [12].

Lemma 3.1. There exists a holomorphic coordinate system centered at $x_{0}$ such that for all $i$ and $j$,

$$
g_{i \bar{j}}\left(x_{0}\right)=\delta_{i j}, \quad \partial_{j} g_{i \bar{i}}\left(x_{0}\right)=0,
$$

and also such that the matrix $\varphi_{i \bar{j}}\left(x_{0}, t_{0}\right)$ is diagonal.

Applying $\triangle^{\prime}-\frac{\partial}{\partial t}$ to $Q$,

$$
\begin{aligned}
\left(\triangle^{\prime}-\frac{\partial}{\partial t}\right) Q= & \frac{\triangle^{\prime} \operatorname{tr}_{g} g^{\prime}}{\operatorname{tr}_{g} g^{\prime}}-\frac{\left|\partial \operatorname{tr}_{g} g^{\prime}\right|_{g^{\prime}}^{2}}{\left(\operatorname{tr}_{g} g^{\prime}\right)^{2}}-\frac{\triangle(\partial \varphi / \partial t)}{\operatorname{tr}_{g} g^{\prime}}+A \frac{\partial \tilde{\varphi}}{\partial t} \mathrm{e}^{A\left(\sup _{M \times[0, T)} \tilde{\varphi}-\tilde{\varphi}\right)} \\
& +\triangle^{\prime} \mathrm{e}^{A\left(\sup _{M \times[0, T)} \tilde{\varphi}-\tilde{\varphi}\right)} .
\end{aligned}
$$


First, we will control the first and third terms in (3.3) simultaneously. We apply the complex Laplacian $\triangle$ to the complex Monge-Ampère equation

$$
\begin{aligned}
\triangle \frac{\partial \varphi}{\partial t}= & -g^{k \bar{l}} g^{\prime p \bar{j}} g^{\prime i \bar{q}} \partial_{k} g_{p \bar{q}}^{\prime} \partial_{\bar{l}} g_{i \bar{j}}^{\prime}+g^{k \bar{l}} g^{\prime i \bar{j}} \partial_{k} \partial_{\bar{l}} g_{i \bar{j}}^{\prime}+g^{k \bar{l}} g^{p \bar{j}} g^{i \bar{q}} \partial_{k} g_{p \bar{q}} \partial_{\bar{l}} g_{i \bar{j}} \\
& -g^{k \bar{l}} g^{i \bar{j}} \partial_{k} \partial_{\bar{l}} g_{i \bar{j}}-\triangle F \\
= & \sum_{i, k} g^{\prime i \bar{i}} \varphi_{i \bar{i} k \bar{k}}-\sum_{i, j, k} g^{\prime i \bar{i}} g^{\prime j \bar{j}} \partial_{k} g_{i \bar{j}}^{\prime} \partial_{\bar{k}} g_{j \bar{i}}^{\prime}+E_{1} .
\end{aligned}
$$

For the first term in (3.3), following a calculation in [25] (see Equation (2.6) in $[25])$ gives:

$$
\triangle^{\prime} \operatorname{tr}_{g} g^{\prime}=\sum_{i, k} g^{\prime i \bar{i}} \varphi_{i \bar{i} k \bar{k}}-2 \operatorname{Re}\left(\sum_{i, j, k} g^{\prime i \bar{i}} \partial_{\bar{i}} g_{j \bar{k}} \varphi_{k \bar{j} i}\right)+E_{2} .
$$

We will now handle the $2 \operatorname{Re}\left(\sum_{i, j, k} g^{\prime i \bar{i}} \partial_{\bar{i}} g_{j \bar{k}} \varphi_{k \overline{j i}}\right)$ term in (3.6) using a trick from [12]. Using Lemma 3.2, at the point $\left(x_{0}, t_{0}\right)$,

$$
\sum_{i, j, k} g^{\prime i \bar{i}} \partial_{\bar{i}} g_{j \bar{k}} \varphi_{k \bar{j} i}=\sum_{i} \sum_{j \neq k} g^{\prime i \bar{i}} \partial_{\bar{i}} g_{j \bar{k}} \partial_{k} g_{i \bar{j}}^{\prime}+E_{1}
$$

Hence,

$$
\begin{aligned}
\left|2 \operatorname{Re}\left(\sum_{i, j, k} g^{\prime i \bar{i}} \partial_{\bar{i}} g_{j \bar{k}} \varphi_{k \bar{j} i}\right)\right| \leq & \sum_{i} \sum_{j \neq k} g^{\prime i \bar{i}} g^{\prime \prime \bar{j}} \partial_{k} g_{i \bar{j}}^{\prime} \partial_{\bar{k}} g_{j \bar{i}}^{\prime} \\
& +\sum_{i} \sum_{j \neq k} g^{\prime i \bar{i}} g_{j \bar{j}}^{\prime} \partial_{\bar{i}} g_{j \bar{k}} \partial_{i} g_{k \bar{j}}+E_{1} \\
\leq & \sum_{i} \sum_{j \neq k} g^{\prime i \bar{i}} g^{\prime j \bar{j}} \partial_{k} g_{i \bar{j}}^{\prime} \partial_{\bar{k}} g_{j \bar{i}}^{\prime}+E_{2} .
\end{aligned}
$$

Putting together (3.5), (3.6), and (3.8) gives

$$
\triangle^{\prime} \operatorname{tr}_{g} g^{\prime}-\triangle \frac{d \varphi}{d t} \geq \sum_{i, j} g^{\prime i \bar{i}} g^{\prime j \bar{j}} \partial_{j} g_{i \bar{j}}^{\prime} \partial_{\bar{j}} g_{j \bar{i}}^{\prime}+E_{2}
$$

Now we will control the $\frac{\left|\partial \operatorname{tr}_{g} g^{\prime}\right|_{g^{\prime}}^{2}}{\left(\operatorname{tr}_{g} g^{\prime}\right)^{2}}$ term in (3.3). By Lemma 3.2 we have at $\left(x_{0}, t_{0}\right)$,

$$
\partial_{i} \operatorname{tr}_{g} g^{\prime}=\partial_{i} \triangle \varphi=\partial_{i} \sum_{j} \varphi_{j \bar{j}}=\sum_{j} \partial_{j} \varphi_{i \bar{j}}=\sum_{j} \partial_{j} g_{i \bar{j}}^{\prime}-\sum_{j} \partial_{j} g_{i \bar{j}} .
$$


So

(3.11)

$$
\frac{\left|\partial \operatorname{tr}_{g} g^{\prime}\right|_{g^{\prime}}^{2}}{\operatorname{tr}_{g} g^{\prime}}=\frac{1}{\operatorname{tr}_{g} g^{\prime}} \sum_{i, j, k} g^{\prime i \bar{i}} \partial_{j} g_{i \bar{j}}^{\prime} \partial_{\bar{k}} g_{k \bar{i}}^{\prime}-\frac{2}{\operatorname{tr}_{g} g^{\prime}} \operatorname{Re}\left(\sum_{i, j, k} g^{\prime i \bar{i}} \partial_{j} g_{i \bar{j}} \partial_{\bar{k}} g_{k \bar{i}}^{\prime}\right)+E_{1}
$$

As in Yau's second-order estimate, we use Cauchy-Schwarz on the first term in (3.11) (see [25] Equation (2.15) for the exact calculation).

$$
\frac{1}{\operatorname{tr}_{g} g^{\prime}} \sum_{i, j, k} g^{\prime i \bar{i}} \partial_{j} g_{i \bar{j}}^{\prime} \partial_{\bar{k}} g_{k \bar{i}}^{\prime} \leq \sum_{i, j} g^{\prime i \bar{i}} g^{\prime j \bar{j}} \partial_{j} g_{i \bar{j}}^{\prime} \partial_{\bar{j}} g_{j \bar{i}}^{\prime}
$$

To deal with the second term in $(3.11)$, since $\left(x_{0}, t_{0}\right)$ is the maximum point of $Q, \partial_{\bar{i}} Q=0$ implies

$$
\frac{1}{\operatorname{tr}_{g} g^{\prime}} \sum_{k} \partial_{\bar{i}} g_{k \bar{k}}^{\prime}=A \partial_{\bar{i}} \varphi \mathrm{e}^{A\left(\sup _{M \times[0, T)} \tilde{\varphi}-\tilde{\varphi}\right)} .
$$

Using Equations (3.13) and (3.10) we can bound the difficult term

$$
\begin{aligned}
& \left|\frac{2}{\operatorname{tr}_{g} g^{\prime}} \operatorname{Re}\left(\sum_{i, j, k} g^{\prime i \bar{i}} \partial_{j} g_{i \bar{j}} \partial_{\bar{k}} g_{k \bar{i}}^{\prime}\right)\right| \\
& \quad=\left|\frac{A}{\operatorname{tr}_{g} g^{\prime}} e^{A\left(\sup _{M \times[0, T)} \tilde{\varphi}-\tilde{\varphi}\right)} 2 \operatorname{Re}\left(\sum_{i, j, k} g^{\prime i \bar{i}} \partial_{j} g_{i \bar{j}} \partial_{\bar{i}} \varphi\right)\right|+E_{1} \\
& \leq A^{2}|\partial \varphi|_{g^{\prime}}^{2} e^{A\left(\sup _{M \times[0, T)} \tilde{\varphi}-\tilde{\varphi}\right)}+\frac{C\left(\operatorname{tr}_{g^{\prime}} g\right)}{\left(\operatorname{tr}_{g} g^{\prime}\right)^{2}} e^{A\left(\sup _{M \times[0, T)} \tilde{\varphi}-\tilde{\varphi}\right)}+E_{1} \\
& \leq A^{2}|\partial \varphi|_{g^{\prime}}^{2} e^{A\left(\sup _{M \times[0, T)} \tilde{\varphi}-\tilde{\varphi}\right)}+C\left(\operatorname{tr}_{g^{\prime}} g\right) e^{A\left(\sup _{M \times[0, T)} \tilde{\varphi}-\tilde{\varphi}\right)}+E_{1},
\end{aligned}
$$

where for the last inequality we used the fact that $\operatorname{tr}_{g} g^{\prime}$ is bounded from below away from zero by the flow Equation (1.1) and estimate (2.1).

Plugging (3.12) and (3.14) into (3.11) gives

$$
\begin{aligned}
& \frac{\left|\partial \operatorname{tr}_{g} g^{\prime}\right|_{g^{\prime}}^{2}}{\left(\operatorname{tr}_{g} g^{\prime}\right)^{2}} \leq \frac{1}{\left(\operatorname{tr}_{g} g^{\prime}\right)^{2}} \sum_{i, j} g^{\prime i \bar{i}} g^{\prime j \bar{j}} \partial_{j} g_{i \bar{j}}^{\prime} \partial_{\bar{j}} g_{j \bar{i}}^{\prime}+A^{2}|\partial \varphi|_{g^{\prime}}^{2} \mathrm{e}^{A\left(\sup _{M \times[0, T)} \tilde{\varphi}-\tilde{\varphi}\right)} \\
& +C\left(\operatorname{tr}_{g^{\prime}} g\right) \mathrm{e}^{A\left(\sup _{M \times[0, T)} \tilde{\varphi}-\tilde{\varphi}\right)}+E_{1} .
\end{aligned}
$$


Combining (3.9) and (3.15) with (3.3) at the point $\left(x_{0}, t_{0}\right)$, we obtain the inequality

$$
\begin{aligned}
0 \geq & \frac{1}{\operatorname{tr}_{g} g^{\prime}}\left(\sum_{i, j} g^{\prime i \bar{i}} g^{\prime j \bar{j}} \partial_{j} g_{i \bar{j}}^{\prime} \partial_{\bar{j}} g_{j \bar{i}}^{\prime}+E_{2}\right)-\frac{1}{\operatorname{tr}_{g} g^{\prime}} \sum_{i, j} g^{\prime i \bar{i}} g^{\prime j \bar{j}} \partial_{j} g_{i \bar{j}}^{\prime} \partial_{\bar{j}} g_{j \bar{i}}^{\prime} \\
& -A^{2}|\partial \varphi|_{g^{\prime}}^{2} \mathrm{e}^{A\left(\sup _{M \times[0, T)} \tilde{\varphi}-\tilde{\varphi}\right)}-\operatorname{tr}_{g^{\prime}} g \mathrm{e}^{A\left(\sup _{M \times[0, T)} \tilde{\varphi}-\tilde{\varphi}\right)}+E_{1} \\
& +A \frac{\partial \tilde{\varphi}}{\partial t} \mathrm{e}^{A\left(\sup _{M \times[0, T)} \tilde{\varphi}-\tilde{\varphi}\right)}+\left(-A n+A \operatorname{tr}_{g^{\prime}} g+A^{2}|\partial \varphi|_{g^{\prime}}^{2}\right) \mathrm{e}^{A\left(\sup _{M \times[0, T)} \tilde{\varphi}-\tilde{\varphi}\right)} \\
\geq & -A(C+n) \mathrm{e}^{A\left(\sup _{M \times[0, T)} \tilde{\varphi}-\tilde{\varphi}\right)}+(A-1) \operatorname{tr}_{g^{\prime}} g \mathrm{e}^{A\left(\sup _{M \times[0, T)} \tilde{\varphi}-\tilde{\varphi}\right)}-C_{1} \operatorname{tr}_{g^{\prime}} g \\
\geq & -A(C+n) \mathrm{e}^{A\left(\sup _{M \times[0, T)} \tilde{\varphi}-\tilde{\varphi}\right)}+\left(A-1-C_{1}\right) \operatorname{tr}_{g^{\prime}} g .
\end{aligned}
$$

Taking $A$ large enough so that

$$
\left(A-1-C_{1}\right)>0
$$

implies that at $\left(x_{0}, t_{0}\right)$,

$$
\operatorname{tr}_{g^{\prime}} g\left(x_{0}, t_{0}\right) \leq C \mathrm{e}^{A\left(\sup _{M \times[0, T)} \tilde{\varphi}-\inf _{M \times[0, T)} \tilde{\varphi}\right)} .
$$

Then

$$
\begin{aligned}
\operatorname{tr}_{g} g^{\prime}\left(x_{0}, t_{0}\right) & \leq \frac{1}{(n-1) !}\left(\operatorname{tr}_{g^{\prime}} g\right)^{n-1} \frac{\operatorname{det} g^{\prime}}{\operatorname{det} g} \\
& =\frac{1}{(n-1) !}\left(\operatorname{tr}_{g^{\prime}} g\right)^{n-1} \mathrm{e}^{F-\frac{\partial \varphi}{\partial t}} \\
& \leq C \mathrm{e}^{A(n-1)\left(\sup _{M \times[0, T)} \tilde{\varphi}-\inf _{M \times[0, T)} \tilde{\varphi}\right)} .
\end{aligned}
$$

For all $(x, t)$ in $M \times\left[0, t^{\prime}\right]$

$$
\begin{aligned}
& \log \operatorname{tr}_{g} g^{\prime}(x, t)+\mathrm{e}^{A\left(\sup _{M \times[0, T)} \tilde{\varphi}-\tilde{\varphi}(x, t)\right)} \leq \log \left(C \mathrm{e}^{A(n-1)\left(\sup _{M \times[0, T)} \tilde{\varphi}-\inf _{M \times[0, T)} \tilde{\varphi}\right)}\right) \\
& +\mathrm{e}^{A\left(\sup _{M \times[0, T)} \tilde{\varphi}-\inf _{M \times[0, T)} \tilde{\varphi}\right)} \\
& \operatorname{tr}_{g} g^{\prime} \leq C_{1} \mathrm{e}^{C_{2}\left(\sup _{M \times[0, T)} \tilde{\varphi}-\inf _{M \times[0, T)} \tilde{\varphi}\right)} \\
& \times \mathrm{e}^{\left(\mathrm{e}^{A\left(\sup _{M \times[0, T)} \tilde{\varphi}-\inf _{M \times[0, T)} \tilde{\varphi}\right)}-\mathrm{e}^{A\left(\sup _{M \times[0, T)} \tilde{\varphi}-\tilde{\varphi}\right)}\right)} .
\end{aligned}
$$




\section{The Hölder estimate for the metric}

The estimate in this section is local, so it suffices to work in a domain in $\mathbb{C}^{n}$. To fix some notation, define the parabolic distance function between two points $\left(x, t_{1}\right)$ and $\left(y, t_{2}\right)$ in $\mathbb{C}^{n} \times[0, T)$ to be $\left|\left(x, t_{1}\right)-\left(y, t_{2}\right)\right|=\max (\mid x-$ $\left.y|,| t_{1}-\left.t_{2}\right|^{1 / 2}\right)$.

For a domain $\Omega \in \mathbb{C}^{n} \times[0, T)$ and a real number $\alpha \in(0,1)$, define for a function $\varphi$ on $\mathbb{C}^{n} \times[0, T)$,

$$
[\varphi]_{\alpha,\left(x_{0}, t_{0}\right)}=\sup _{(x, t) \in \Omega \backslash\left\{\left(x_{0}, t_{0}\right)\right\}} \frac{\left|\varphi(x, t)-\varphi\left(x_{0}, t_{0}\right)\right|}{\left|(x, t)-\left(x_{0}, t_{0}\right)\right|^{\alpha}}
$$

and

$$
[\varphi]_{\alpha, \Omega}=\sup _{(x, t) \in \Omega}[\varphi]_{\alpha,(x, t)}
$$

We will show that

$$
\left[g_{i \bar{j}}^{\prime}\right]_{\alpha, \Omega} \leq C,
$$

for an appropriate choice of $\Omega$. The smoothness of $\varphi$ and $\tilde{\varphi}$ will follow. Given the Hölder bound for the metric and the second-order estimate for $\tilde{\varphi}$, we can differentiate the flow and apply Schauder estimates to achieve higher regularity.

Theorem 4.1. Let $\varphi$ be a solution to the flow (1.1) and $g_{i \bar{j}}^{\prime}=g_{i \bar{j}}+\varphi_{i \bar{j}}$. Fix $\varepsilon>0$. Then there exists $\alpha \in(0,1)$ and a constant $C$ depending only on the initial data and $\varepsilon$ such that

$$
\left[g_{i \bar{j}}^{\prime}\right]_{\alpha, \Omega} \leq C
$$

where $\Omega=M \times[\varepsilon, T)$.

We apply a method due to Evans [10] and Krylov [16]. The proof itself is essentially contained in [17] and [13], but only in the case where the manifold is $\mathbb{R}^{n}$. Hence, we produce a self-contained proof in the notation of this problem. The method of this proof follows closely with the analogous estimate in $[22,25]$. The main issue is applying the correct Harnack inequality to get the estimate.

Proof. Let $B \in \mathbb{C}^{n}$ be an open ball about the origin. Fix a point $t_{0} \in[\varepsilon, T)$. To prove (4.2) it suffices to show that for sufficiently small $R>0$ there exists 
a uniform $C$ and $\delta>0$ such that

$$
\sum_{i=1}^{n} \operatorname{osc}_{Q(R)}\left(\varphi_{\gamma_{i} \bar{\gamma}_{i}}\right)+\operatorname{osc}_{Q(R)}\left(\varphi_{t}\right) \leq C R^{\delta}
$$

where $\left\{\gamma_{i}\right\}$ is a basis for $\mathbb{C}^{n}$ and $Q(R)$ is the parabolic cylinder

$$
Q(R)=\left\{(x, t) \in B \times[0, T)|| x \mid \leq R, t_{0}-R^{2} \leq t \leq t_{0}\right\}
$$

We rewrite the flow as

$$
\frac{\partial \varphi}{\partial t}=\log \operatorname{det} g_{i \bar{j}}^{\prime}+H
$$

where $H=-\log \operatorname{det} g_{i \bar{j}}-F$. We define the operator $\Phi$ on a matrix $A$ by

$$
\Phi(A)=\log \operatorname{det} A
$$

then (4.3) becomes

$$
\frac{\partial \varphi}{\partial t}=\Phi\left(g^{\prime}\right)+H
$$

By the concavity of $\Phi$, for all $\left(x, t_{1}\right)$ and $\left(y, t_{2}\right)$ in $B \times[0, T)$,

$$
\begin{gathered}
\sum \frac{\partial \Phi}{\partial a_{i \bar{j}}}\left(g^{\prime}\left(y, t_{2}\right)\right)\left(g_{i \bar{j}}^{\prime}\left(x, t_{1}\right)-g_{i \bar{j}}^{\prime}\left(y, t_{2}\right)\right) \\
\geq \frac{\partial \varphi}{\partial t}\left(x, t_{1}\right)-\frac{\partial \varphi}{\partial t}\left(y, t_{2}\right)-H(x)+H(y) .
\end{gathered}
$$

The mean value theorem applied to $H$ shows that

$$
\begin{aligned}
& \frac{\partial \varphi}{\partial t}\left(x, t_{1}\right)-\frac{\partial \varphi}{\partial t}\left(y, t_{2}\right)+\sum \frac{\partial \Phi}{\partial a_{i \bar{j}}}\left(g^{\prime}\left(y, t_{2}\right)\right)\left(g_{i \bar{j}}^{\prime}\left(y, t_{2}\right)-g_{i \bar{j}}^{\prime}\left(x, t_{1}\right)\right) \\
& \quad \leq C|x-y| .
\end{aligned}
$$

Now we must recall a lemma from linear algebra.

Lemma 4.1. There exists a finite number $N$ of unit vectors $\gamma_{\nu}=\left(\gamma_{\nu 1}, \ldots\right.$, $\left.\gamma_{\nu n}\right) \in \mathbb{C}^{n}$ and real-valued functions $\beta_{\nu}$ on $B \times[0, T)$, for $\nu=1,2, \ldots, N$ 
with

(i) $0<C_{1} \leq \beta_{\nu} \leq C_{2}$

(ii) $\gamma_{1}, \ldots, \gamma_{N}$ containing an orthonormal basis of $\mathbb{C}^{n}$

such that

$$
\frac{\partial \Phi}{\partial a_{i \bar{j}}}\left(g^{\prime}\left(y, t_{2}\right)\right)=\sum_{\nu=1}^{N} \beta_{\nu}\left(y, t_{2}\right) \gamma_{\nu i} \overline{\gamma_{\nu j}}
$$

We define for $\nu=1, \ldots, N$,

$$
w_{\nu}=\partial_{\gamma_{\nu}} \partial_{\overline{\gamma_{\nu}}} \varphi=\sum_{i, j=1}^{n} \gamma_{\nu i} \overline{\gamma_{\nu j}} \varphi_{i \bar{j}}
$$

We write $w_{0}=-\frac{\partial \varphi}{\partial t}$ and $\beta_{0}=1$. Then using the linear algebra lemma, (4.5) can be rewritten as

$$
\sum_{\nu=0}^{N} \beta_{\nu}\left(y, t_{2}\right)\left(w_{\nu}\left(y, t_{2}\right)-w_{\nu}\left(x, t_{1}\right)\right) \leq C|x-y| .
$$

Letting $\gamma$ be an arbitrary unit vector in $\mathbb{C}^{n}$, we differentiate the flow (1.1) along $\gamma$ and $\bar{\gamma}$ :

$$
\begin{aligned}
\frac{\partial \varphi_{\gamma \bar{\gamma}}}{\partial t} & =\frac{\partial^{2} \Phi}{\partial a_{i \bar{j}} \partial a_{k \bar{l}}}\left(g^{\prime}\right) g_{i \bar{j} \gamma}^{\prime} g_{k \bar{l} \bar{\gamma}}^{\prime}+\frac{\partial \Phi}{\partial a_{i \bar{j}}}\left(g^{\prime}\right) g_{i \bar{j} \gamma \bar{\gamma}}^{\prime}+H_{\gamma \bar{\gamma}} \\
& \leq g^{\prime i \bar{j}} g_{i \bar{j} \gamma \bar{\gamma}}^{\prime}+H_{\gamma \bar{\gamma}}
\end{aligned}
$$

where on the last line we used the concavity of $\Phi$ and the fact that $\frac{\partial \Phi}{\partial a_{i \bar{j}}}\left(g^{\prime}\right)=$ $g^{\prime i \bar{j}}$. Applying $\frac{\partial}{\partial t}$ to (4.4) gives

$$
\frac{\partial \varphi_{t}}{\partial t}=g^{\prime i \bar{j}} \varphi_{i \bar{j} t}
$$

From (4.8) and (4.9) we have a bounded function $h$ (depending on $g^{\prime i \bar{j}}$ which is bounded by Theorem 3.1) such that

$$
-\frac{\partial w_{\nu}}{\partial t}+g^{\prime i \bar{j}} \partial_{i} \partial_{\bar{j}} w_{\nu} \geq h .
$$


Recall that $t_{0}$ is a fixed point in $[\varepsilon, T)$. Pick $R>0$ small enough such that $t_{0}-5 R^{2}>t_{0} / 2$. We define another parabolic cylinder

$$
\Theta(R)=\left\{(x, t) \in B \times[0, T)|| x \mid<R, t_{0}-5 R^{2} \leq t \leq t_{0}-4 R^{2}\right\}
$$

For $s=1,2$ and $\nu=0,1, \ldots, N$, let

$$
M_{s \nu}=\sup _{Q(s R)} w_{\nu}, m_{s \nu}=\inf _{Q(s R)} w_{\nu}
$$

and

$$
\psi(s R)=\sum_{\nu=0}^{N}\left(M_{s \nu}-m_{s \nu}\right)
$$

We let $l$ be an integer such that $0 \leq l \leq N$ and $v=M_{2 l}-w_{l}$. To continue we need Theorem 7.37 from [17]. We say that $v \in W_{2 n+1}^{2,1}$ if $v_{x}, v_{i j}, v_{i \bar{j}}, v_{\overline{i j}}$, and $v_{t}$ are in $L^{2 n+1}$. We restate the theorem as follows.

Lemma 4.2. Suppose that $v(x, t) \in W_{2 n+1}^{2,1}$ satisfies

$$
-\frac{\partial v}{\partial t}+g^{\prime i \bar{j}} \partial_{i} \partial_{\bar{j}} v \leq f
$$

and $v \geq 0$ on $Q(4 R)$. Then there exists a constant $C$ and $a p>0$ depending only on the bounds of $g^{\prime i \bar{j}}$ and the eigenvalues of $g^{\prime i \bar{j}}$ such that

$$
\frac{1}{R^{2 n+2}}\left(\int_{\Theta(R)} v^{p}\right)^{1 / p} \leq C\left(\inf _{Q(R)} v+R^{2 n / 2 n+1}\|f\|_{n+1}\right) .
$$

Since $v$ satisfies $-\frac{\partial v}{\partial t}+g^{\prime i \bar{j}} \partial_{i} \partial_{\bar{j}} v \leq-h$, we can apply the Harnack inequality to obtain

$$
\frac{1}{R^{2 n+2}}\left(\int_{\Theta(R)}\left(M_{2 l}-w_{l}\right)^{p}\right)^{1 / p} \leq C\left(M_{2 l}-M_{l}+R^{2 n / 2 n+1}\right) .
$$

For every $\left(x, t_{1}\right)$ and $\left(y, t_{2}\right)$ in $Q(2 R),(4.7)$ gives

$$
\beta_{l}\left(y, t_{2}\right)\left(w_{l}\left(y, t_{2}\right)-w_{l}\left(x, t_{1}\right)\right) \leq C R+\sum_{\nu \neq l} \beta_{\nu}\left(w_{\nu}\left(x, t_{1}\right)-w_{\nu}\left(y, t_{2}\right)\right) .
$$


The definition of $m_{2 l}$ allows us to choose $\left(x, t_{1}\right)$ in $Q(2 R)$ such that $w_{l}\left(x, t_{1}\right) \leq m_{2 l}+\varepsilon$. Since $\varepsilon$ is arbitrary,

$$
w_{l}\left(y, t_{2}\right)-m_{2 l} \leq C R+C_{2} \sum_{\nu \neq l}\left(M_{2 \nu}-w_{\nu}\left(y, t_{2}\right)\right) .
$$

After integrating over $\Theta(R)$ and applying (4.11), we have

$$
\begin{aligned}
& \frac{1}{R^{2 n+2}}\left(\int_{\Theta(R)}\left(w_{l}-m_{2 l}\right)^{p}\right)^{1 / p} \\
& \leq \frac{1}{R^{2 n+2}}\left(\int_{\Theta(R)}\left(C R+C_{2} \sum_{\nu \neq l}\left(M_{2 \nu}-w_{\nu}\right)\right)^{p}\right)^{1 / p} \\
& \leq C_{3} R+C_{4} \sum_{\nu \neq l} \frac{1}{R^{2 n+2}}\left(\int_{\Theta(R)}\left(M_{2 \nu}-w_{\nu}\right)^{p}\right)^{1 / p} \\
& \leq C_{5} \sum_{\nu \neq l}\left(M_{2 \nu}-M_{\nu}\right)+C_{6} R^{2 n / 2 n+1}
\end{aligned}
$$

where on the last line we used the fact that $R<1$ is small. Adding (4.11) and (4.12) yields

$$
\begin{aligned}
M_{2 l}-m_{2 l} & \leq C_{7} \sum_{\nu=0}^{N}\left(M_{2 \nu}-M_{\nu}\right)+C_{8} R^{2 n / 2 n+1} \\
& \leq C_{7} \sum_{\nu=0}^{N}\left(M_{2 \nu}-M_{\nu}+m_{\nu}-m_{2 \nu}\right)+C_{8} R^{2 n / 2 n+1} \\
& =C_{7}(\psi(2 R)-\psi(R))+C_{8} R^{2 n / 2 n+1} .
\end{aligned}
$$

Summing over $l$ shows that

$$
\psi(2 R) \leq C_{9}(\psi(2 R)-\psi(R))+C_{10} R^{2 n / 2 n+1}
$$

and thus for some $0<\lambda<1$,

$$
\psi(R) \leq \lambda \psi(2 R)+C_{11} R^{2 n / 2 n+1} .
$$

Applying a standard iteration argument (see Chapter 8 in [13]) shows that

$$
\psi(R) \leq C R^{\delta},
$$

for some $\delta>0$, completing the proof. 


\section{Long-time existence and smoothness of the normalized solution}

In this section, we show that the solution $\varphi$ and its normalization $\tilde{\varphi}$ are smooth and exist for all time, hence proving part of the main theorem. The proof uses a standard bootstrapping argument.

Theorem 5.1. Let $(M, g)$ be a Hermitian manifold and $F$ a smooth function on $M$. Let $\varphi$ be a solution to the flow

$$
\frac{\partial \varphi}{\partial t}=\log \frac{\operatorname{det}\left(g_{i \bar{j}}+\varphi_{i \bar{j}}\right)}{\operatorname{det}\left(g_{i \bar{j}}\right)}-F
$$

and let $\tilde{\varphi}=\varphi-\int_{M} \varphi \omega^{n}$. Then there are uniform $C^{\infty}$ estimates for $\tilde{\varphi}$ on $[0, T)$. Moreover, $T=\infty$.

Proof. Differentiating the flow with respect to $z^{k}$ gives

$$
\frac{\partial \varphi_{k}}{\partial t}=g^{\prime i \bar{j}} \partial_{i} \partial_{\bar{j}} \varphi_{k}-F_{k}-\frac{\partial}{\partial z^{k}} \log \operatorname{det} g_{i \bar{j}}
$$

The second-order estimate implies that the above equation is uniformly parabolic. Theorem 4.1 shows that the coefficients in the above equation are Hölder continuous with exponent $\alpha$. Using the Schauder estimate (see Theorem 4.9 in [17], for example) gives a uniform parabolic $C^{2+\alpha}$ bound on $\varphi_{k}$. Similarly, one obtains a uniform parabolic $C^{2+\alpha}$ estimate for $\varphi_{\bar{k}}$.

But the better differentiability of $\varphi$ allows us to differentiate the flow again and obtain a uniformly parabolic equation with Hölder continuous coefficients. The Schauder estimate will give a uniform parabolic $C^{2+\alpha}$ estimate on $\varphi_{k l}, \varphi_{k \bar{l}}$, and $\varphi_{\bar{k} \bar{l}}$. Repeated application shows that $\tilde{\varphi}$ is uniformly bounded in $C^{\infty}$. Hence $\tilde{\varphi}$ and thus $\varphi$ are smooth. We note that $\varphi$ is not necessarily bounded in $C^{0}$. The above iterations only provide regularity for the derivatives of $\varphi$.

To see that $T=\infty$, suppose that for $T<\infty,[0, T)$ is the maximal interval for the existence of the solution. Since $\tilde{\varphi}$ is smooth, we can apply short time existence to extend the flow for $\tilde{\varphi}$ to $[0, T+\varepsilon)$, a contradiction.

\section{The Harnack inequality}

We begin this section by proving lemmas analogous to those of Li and Yau [18] for the equation $\frac{\partial u}{\partial t}=g^{\prime i \bar{j}} \partial_{i} \partial_{\bar{j}} u$ for a positive function $u$ on a Hermitian 
manifold (see [28] for the proof of these lemmas in the Kähler case). The lemmas lead to a Harnack inequality, which in turn shows that the time derivative of $\tilde{\varphi}$ decays exponentially. This allows us to prove the convergence of $\tilde{\varphi}$ as $t$ tends to infinity.

In this section, we again use the notation $u_{t}=\frac{\partial u}{\partial t}$ and $u_{i}=\partial_{i} u$ for the ordinary derivatives of a function $u$ on $M$.

Let $u$ be a positive function on $M$. Consider the heat-type equation

$$
u_{t}=g^{i i \bar{j}} u_{i \bar{j}}
$$

where $g_{i \bar{j}}^{\prime}$ denotes the time dependent metric $g_{i \bar{j}}+\varphi_{i \bar{j}}$. Define $\tilde{\varphi}=\varphi-$ $\int_{M} \varphi \omega^{n}$.

Define $f=\log u$ and $F=t\left(|\partial f|^{2}-\alpha f_{t}\right)$ where $1<\alpha<2$. We remark that this $F$ is different from the one in Equation (1.1). Then

$$
g^{\prime i \bar{j}} f_{i \bar{j}}-f_{t}=-|\partial f|^{2}
$$

where $\partial f$ is the vector containing the ordinary derivatives of $f$ and

$$
|\partial f|^{2}=g^{i \bar{j}} \partial_{i} f \partial_{j} f
$$

Also write

$$
\langle X, Y\rangle=g^{i i \bar{j}} X_{i} Y_{\bar{j}}
$$

for the inner product of two vectors $X$ and $Y$ with respect to $g_{i \bar{j}}^{\prime}$.

We now prove an estimate that will be useful in applying the maximum principle to $F$.

Lemma 6.1. There exist constants $C_{1}$ and $C_{2}$ depending only on the bounds of the metric $g^{\prime}$ such that for $t>0$,

$$
\begin{aligned}
g^{\prime k \bar{l}} F_{k \bar{l}}-F_{t} \geq & \frac{t}{2 n}\left(|\partial f|^{2}-f_{t}\right)^{2}-2 \operatorname{Re}\langle\partial f, \partial F\rangle-\left(|\partial f|^{2}-\alpha f_{t}\right) \\
& -C_{1} t|\partial f|^{2}-C_{2} t .
\end{aligned}
$$

Proof. First calculate $F=-t g^{\prime i \bar{j}} f_{i \bar{j}}-t(\alpha-1) f_{t}$. Then

$$
\left(g^{i i \bar{j}} f_{i \bar{j}}\right)_{t}=\frac{1}{t^{2}} F-\frac{1}{t} F_{t}-(\alpha-1) f_{t t}
$$


and

$$
\begin{aligned}
F_{t} & =|\partial f|^{2}-\alpha f_{t}+t\left(g^{i i \bar{j}} f_{t i} f_{\bar{j}}+g^{\prime i \bar{j}} f_{i} f_{t \bar{j}}+\left(\frac{\partial}{\partial t} g^{i i \bar{j}}\right) f_{i} f_{\bar{j}}-\alpha f_{t t}\right) \\
& =|\partial f|^{2}-\alpha f_{t}+2 t \operatorname{Re}\left\langle\partial f, \partial\left(f_{t}\right)\right\rangle+t\left(\frac{\partial}{\partial t} g^{\prime i \bar{j}}\right) f_{i} f_{\bar{j}}-\alpha t f_{t t} .
\end{aligned}
$$

We calculate $g^{\prime k \bar{l}} F_{k \bar{l}}$ to get the desired estimate.

$$
\begin{aligned}
g^{\prime k \bar{l}} F_{k \bar{l}}= & t g^{\prime k \bar{l}}\left[\left(g^{\prime i \bar{j}}\right)_{k \bar{l}} f_{i} f_{\bar{j}}+\left(g^{\prime i \bar{j}}\right)_{k} f_{i \bar{l}} f_{\bar{j}}+\left(g^{\prime i \bar{j}}\right)_{k} f_{i} f_{\bar{j} \bar{l}}\right. \\
& +\left(g^{\prime i \bar{j}}\right)_{\bar{l}} f_{i k} f_{\bar{j}}+g^{\prime i \bar{j}} f_{i k \bar{l}} f_{\bar{j}}+g^{\prime i \bar{j}} f_{i k} f_{\bar{j} \bar{l}} \\
& \left.+\left(g^{\prime i \bar{j}}\right)_{\bar{l}} f_{i} f_{\bar{j} k}+g^{\prime i \bar{j}} f_{i \bar{l}} f_{\bar{j} k}+g^{\prime i \bar{j}} f_{i} f_{\bar{j} k \bar{l}}-\alpha f_{t k \bar{l}}\right] .
\end{aligned}
$$

Now we control all of the above terms using the bounds on the metric obtained in Theorem 3.1 and the higher-order bounds from Theorem 5.1. For the first term of (6.3),

$$
\left|\operatorname{tg}^{\prime k \bar{l}}\left(g^{\prime i \bar{j}}\right)_{k \bar{l}} f_{i} f_{\bar{j}}\right| \leq C_{1} t|\partial f|^{2} .
$$

Let $\varepsilon>0$. We bound the second and third terms of (6.3) with the inequalities

$$
\left|t g^{\prime k \bar{l}}\left(g^{\prime i \bar{j}}\right)_{k} f_{i \bar{l}} f_{\bar{j}}\right| \leq \frac{t}{\varepsilon}|\partial f|^{2}+t \varepsilon|\partial \bar{\partial} f|^{2}
$$

and

$$
\left|t g^{\prime k \bar{l}}\left(g^{\prime i \bar{j}}\right)_{k} f_{i} f_{\bar{j} \bar{l}}\right| \leq \frac{t}{\varepsilon}|\partial f|^{2}+t \varepsilon\left|D^{2} f\right|^{2}
$$

where

$$
|\partial \bar{\partial} f|^{2}=g^{\prime k \bar{l}} g^{\prime i \bar{j}} f_{i \bar{l}} f_{\bar{j} k}, \quad\left|D^{2} f\right|^{2}=g^{\prime k \bar{l}} g^{\prime i \bar{j}} f_{i k} f_{\bar{j} \bar{l}} .
$$

Term six is equal to $t\left|D^{2} f\right|^{2}$ and term eight equals $t|\partial \bar{\partial} f|^{2}$. The fifth and ninth terms of (6.3) combine to give

$$
\begin{aligned}
t g^{\prime k \bar{l}} g^{\prime i \bar{j}} f_{i k \bar{l}} f_{\bar{j}}+t g^{\prime k \bar{l}} g^{\prime i \bar{j}} f_{i} f_{\bar{j} k \bar{l}}= & 2 t \operatorname{Re}\left\langle\partial f, \partial\left(g^{\prime k \bar{l}} f_{k \bar{l}}\right)\right\rangle-t g^{\prime i \bar{j}}\left(g^{\prime k \bar{l}}\right)_{i} f_{k \bar{l}} f_{\bar{j}} \\
& -t g^{\prime i \bar{j}}\left(g^{\prime k \bar{l}}\right)_{\bar{j}} f_{i} f_{k \bar{l}} \\
\geq & 2 t \operatorname{Re}\left\langle\partial f, \partial\left(g^{\prime k \bar{l}} f_{k \bar{l}}\right)\right\rangle-\frac{t}{\varepsilon}|\partial f|^{2}-t \varepsilon|\partial \bar{\partial} f|^{2}
\end{aligned}
$$


We use the definition of $F$ to show

$$
\begin{aligned}
& t g^{\prime k \bar{l}} g^{\prime i \bar{j}} f_{i k \bar{l}} f_{\bar{j}}+t g^{\prime k \bar{l}} g^{\prime i \bar{j}} f_{i} f_{\bar{j} k \bar{l}} \\
& \quad \geq-2 \operatorname{Re}\langle\partial f, \partial F\rangle-2 t(\alpha-1) \operatorname{Re}\left\langle\partial f, \partial\left(f_{t}\right)\right\rangle-\frac{t}{\varepsilon}|\partial f|^{2}-t \varepsilon|\partial \bar{\partial} f|^{2} .
\end{aligned}
$$

Applying Equation (6.2) to (6.5) gives

$$
\begin{aligned}
& t g^{\prime k \bar{l}} g^{\prime i \bar{j}} f_{i k \bar{l}} f_{\bar{j}}+t g^{\prime k \bar{l}} g^{\prime i \bar{j}} f_{i} f_{\bar{j} k \bar{l}} \\
& \geq-2 \operatorname{Re}\langle\partial f, \partial F\rangle-(\alpha-1) F_{t}+(\alpha-1)\left(|\partial f|^{2}-\alpha f_{t}\right) \\
& \quad+t(\alpha-1)\left(\frac{\partial}{\partial t} g^{\prime i \bar{j}}\right) f_{i} f_{\bar{j}}-t \alpha(\alpha-1) f_{t t}-\frac{t}{\varepsilon}|\partial f|^{2}-t \varepsilon|\partial \bar{\partial} f|^{2} \\
& \geq-2 \operatorname{Re}\langle\partial f, \partial F\rangle-(\alpha-1) F_{t}+(\alpha-1)\left(|\partial f|^{2}-\alpha f_{t}\right) \\
& \quad-C_{2} t|\partial f|^{2}-t \alpha(\alpha-1) f_{t t}-\frac{t}{\varepsilon}|\partial f|^{2}-t \varepsilon|\partial \bar{\partial} f|^{2}
\end{aligned}
$$

The final term of (6.3) becomes, using (6.1)

$$
\begin{aligned}
-\alpha t g^{\prime k \bar{l}} f_{t k \bar{l}} & =\alpha t\left(\frac{\partial}{\partial t} g^{\prime k \bar{l}}\right) f_{k \bar{l}}-\alpha t \frac{\partial}{\partial t}\left(g^{\prime k \bar{l}} f_{k \bar{l}}\right) \\
& \geq-\frac{C t}{\varepsilon}-t \varepsilon|\partial \bar{\partial} f|^{2}-\frac{\alpha}{t} F+\alpha F_{t}+t \alpha(\alpha-1) f_{t t}
\end{aligned}
$$

We put all of the above in to (6.3), which shows that

$$
\begin{aligned}
g^{\prime k \bar{l}} F_{k \bar{l}} \geq & F_{t}-2 \operatorname{Re}\langle\partial f, \partial F\rangle-\left(|\partial f|^{2}-\alpha f_{t}\right)+t(1-4 \varepsilon)|\partial \bar{\partial} f|^{2} \\
& +t(1-2 \varepsilon)\left|D^{2} f\right|^{2}-t\left(C_{1}+C_{2}+\frac{6}{\varepsilon}\right)|\partial f|^{2}-\frac{C t}{\varepsilon}
\end{aligned}
$$

Taking $\varepsilon$ sufficiently small and applying the arithmetic-geometric mean inequality

$$
|\partial \bar{\partial} f|^{2} \geq \frac{1}{n}\left(g^{\prime k \bar{l}} f_{k \bar{l}}\right)^{2}=\frac{1}{n}\left(|\partial f|^{2}-f_{t}\right)^{2}
$$

we see that

$$
\begin{aligned}
g^{\prime k \bar{l}} F_{k \bar{l}}-F_{t} \geq & \frac{t}{2 n}\left(|\partial f|^{2}-f_{t}\right)^{2}-2 \operatorname{Re}\langle\partial f, \partial F\rangle-\left(|\partial f|^{2}-\alpha f_{t}\right) \\
& -C t|\partial f|^{2}-C t
\end{aligned}
$$

Using the previous lemma, we derive an estimate which will be used to prove the Harnack inequality. 
Lemma 6.2. There exist constants $C_{1}$ and $C_{2}$ depending only on the bounds of the metric $g^{\prime}$ such that for $t>0$,

$$
|\partial f|^{2}-\alpha f_{t} \leq C_{1}+\frac{C_{2}}{t}
$$

Proof. Fix $T>0$ and let $\left(x_{0}, t_{0}\right)$ in $M \times[0, T]$ be where $F$ attains its maximum. Note that we can take $t_{0}>0$. Then at $\left(x_{0}, t_{0}\right)$, from the previous lemma,

$$
\frac{t_{0}}{2 n}\left(|\partial f|^{2}-f_{t}\right)^{2}-\left(|\partial f|^{2}-\alpha f_{t}\right) \leq C_{1} t_{0}|\partial f|^{2}+C_{2} t_{0}
$$

First, we assume that $f_{t}\left(x_{0}, t_{0}\right) \geq 0$, then the $\alpha$ in the above inequality can be dropped to give

$$
\frac{t_{0}}{2 n}\left(|\partial f|^{2}-f_{t}\right)^{2}-\left(|\partial f|^{2}-f_{t}\right) \leq C_{1} t_{0}|\partial f|^{2}+C_{2} t_{0}
$$

We factor the above to obtain

$$
\frac{1}{2 n}\left(|\partial f|^{2}-f_{t}\right)\left(|\partial f|^{2}-f_{t}-\frac{2 n}{t_{0}}\right) \leq C_{1}|\partial f|^{2}+C_{2}
$$

Hence,

$$
|\partial f|^{2}-f_{t} \leq C_{3}|\partial f|+C_{4}+\frac{C_{5}}{t_{0}} .
$$

There exists a constant $C_{6}$ such that

$$
C_{3}|\partial f| \leq\left(1-\frac{1}{\alpha}\right)|\partial f|^{2}+C_{6}
$$

We plug this in to the previous inequality, showing that

$$
\frac{1}{\alpha}|\partial f|^{2}-f_{t} \leq C_{7}+\frac{C_{5}}{t_{0}} .
$$

At the point $\left(x_{0}, t_{0}\right)$, we have

$$
F\left(x_{0}, t_{0}\right)=t_{0}\left(|\partial f|^{2}\left(x_{0}, t_{0}\right)-\alpha f_{t}\left(x_{0}, t_{0}\right)\right) \leq C_{8} t_{0}+C_{5} .
$$

Hence for all $x$ in $M$,

$$
\begin{aligned}
F(x, T) & \leq F\left(x_{0}, t_{0}\right) \\
& \leq C_{8} t_{0}+C_{5} \\
& \leq C_{8} T+C_{5}
\end{aligned}
$$

completing the proof for this case. 
Now we consider the case where $f_{t}\left(x_{0}, t_{0}\right)<0$. Using (6.6) at the point $\left(x_{0}, t_{0}\right)$,

$$
\frac{t_{0}}{2 n}|\partial f|^{4}-|\partial f|^{2} \leq C_{1} t_{0}|\partial f|^{2}+C_{2} t_{0}-\alpha f_{t} .
$$

We factor the above to obtain

$$
|\partial f|^{2}\left(\frac{1}{2 n}|\partial f|^{2}-\frac{1}{t_{0}}-C_{1}\right) \leq C_{2}-\frac{\alpha}{t_{0}} f_{t} .
$$

Hence,

$$
|\partial f|^{2} \leq C_{3}+\frac{C_{4}}{t_{0}}-\frac{1}{2} f_{t} .
$$

We use (6.6) again and the condition that $f_{t}\left(x_{0}, t_{0}\right)<0$ to see that

$$
\frac{t_{0}}{2 n} f_{t}^{2}+\alpha f_{t} \leq C_{1} t_{0}|\partial f|^{2}+|\partial f|^{2}+C_{2} t_{0} .
$$

By factoring the above, we show that

$$
\frac{1}{2 n}\left(-f_{t}\right)\left(-f_{t}-\frac{2 n \alpha}{t_{0}}\right) \leq C_{1}|\partial f|^{2}+\frac{1}{t_{0}}|\partial f|^{2}+C_{2} .
$$

And so

$$
-f_{t} \leq C_{5}+\frac{C_{6}}{t_{0}}+\frac{1}{2}|\partial f|^{2} .
$$

We plug (6.9) into (6.8), arriving at

$$
|\partial f|^{2} \leq C_{3}+\frac{C_{4}}{t_{0}}+\frac{C_{5}}{2}+\frac{C_{6}}{2 t_{0}}+\frac{1}{4}|\partial f|^{2} .
$$

This provides the following estimate for $|\partial f|^{2}$ :

$$
|\partial f|^{2} \leq C_{7}+\frac{C_{8}}{t_{0}} .
$$

Similarly, we can show that

$$
-\alpha f_{t} \leq C_{9}+\frac{C_{10}}{t_{0}} .
$$

We add (6.10) and (6.11) to obtain the estimate

$$
|\partial f|^{2}-\alpha f_{t} \leq C_{11}+\frac{C_{12}}{t_{0}} .
$$


Repeating the argument after (6.7) completes this case and hence the proof.

We use the previous lemma to derive a Harnack inequality similar to that of Li and Yau in the case of a Hermitian manifold.

Lemma 6.3. For $0<t_{1}<t_{2}$,

$$
\sup _{x \in M} u\left(x, t_{1}\right) \leq \inf _{x \in M} u\left(x, t_{2}\right)\left(\frac{t_{2}}{t_{1}}\right)^{C_{2}} \exp \left(\frac{C_{3}}{t_{2}-t_{1}}+C_{1}\left(t_{2}-t_{1}\right)\right),
$$

where $C_{1}, C_{2}$ and $C_{3}$ are constants depending only on the bounds of the metric $g^{\prime}$.

Proof. Let $x, y \in M$, and define $\gamma$ to be the minimal geodesic (with respect to the initial metric $\left.g_{i \bar{j}}\right)$ with $\gamma(0)=y$ and $\gamma(1)=x$. Define a path $\zeta:[0,1] \rightarrow$ $M \times\left[t_{1}, t_{2}\right]$ by $\zeta(s)=\left(\gamma(s),(1-s) t_{2}+s t_{1}\right)$. Then using Lemma 6.2,

$$
\begin{aligned}
\log \frac{u\left(x, t_{1}\right)}{u\left(y, t_{2}\right)}= & \int_{0}^{1} \frac{d}{d s} f(\zeta(s)) d s \\
= & \int_{0}^{1}\left(\langle\dot{\gamma}, 2 \partial f\rangle-\left(t_{2}-t_{1}\right) f_{t}\right) d s \\
\leq & \int_{0}^{1}-\frac{t_{2}-t_{1}}{\alpha}\left(|\partial f|-\frac{\alpha|\dot{\gamma}|}{\left(t_{2}-t_{1}\right)}\right)^{2}+\frac{\alpha|\dot{\gamma}|^{2}}{\left(t_{2}-t_{1}\right)} \\
& +C_{1}\left(t_{2}-t_{1}\right)+C_{2} \frac{t_{2}-t_{1}}{t} d s \\
\leq & \int_{0}^{1} \frac{C_{19}}{t_{2}-t_{1}}+C_{17}\left(t_{2}-t_{1}\right)+C_{18} \frac{t_{2}-t_{1}}{t} d s \\
= & \frac{C_{3}}{t_{2}-t_{1}}+C_{1}\left(t_{2}-t_{1}\right)+C_{2} \log \left(\frac{t_{2}}{t_{1}}\right) .
\end{aligned}
$$

Exponentiating both sides completes the proof.

\section{Convergence of the flow}

With the Harnack inequality, we complete the proof of the main theorem by showing the convergence of $\tilde{\varphi}$ (cf. [4]). 
Proof. Define $u=\frac{\partial \varphi}{\partial t}$. Then

$$
\frac{\partial u}{\partial t}=g^{i \bar{j}} \partial_{i} \partial_{\bar{j}} u
$$

Let $m$ be a positive integer and define

$$
\begin{aligned}
& \xi_{m}(x, t)=\sup _{y \in M} u(y, m-1)-u(x, m-1+t), \\
& \psi_{m}(x, t)=u(x, m-1+t)-\inf _{y \in M} u(y, m-1) .
\end{aligned}
$$

These functions satisfy the heat-type equations

$$
\begin{aligned}
\frac{\partial \xi_{m}}{\partial t} & =g^{\prime i \bar{j}}(m-1+t) \partial_{i} \partial_{\bar{j}} \xi_{m} \\
\frac{\partial \psi_{m}}{\partial t} & =g^{\prime i \bar{j}}(m-1+t) \partial_{i} \partial_{\bar{j}} \psi_{m}
\end{aligned}
$$

First consider the case where $u(x, m-1)$ is not constant. Then $\xi_{m}$ is positive for some $x$ in $M$ at time $t=0$. By the maximum principle, $\xi_{m}$ must be positive for all $x$ in $M$ when $t>0$. Similarly, $\psi_{m}$ is positive everywhere when $t>0$. Hence, we can apply Lemma (6.3) with $t_{1}=\frac{1}{2}$ and $t_{2}=1$ to obtain

$$
\begin{aligned}
& \sup _{x \in M} u(x, m-1)-\inf _{x \in M} u\left(x, m-\frac{1}{2}\right) \leq C\left(\sup _{x \in M} u(x, m-1)-\sup _{x \in M} u(x, m)\right), \\
& \sup _{x \in M} u\left(x, m-\frac{1}{2}\right)-\inf _{x \in M} u(x, m-1) \leq C\left(\inf _{x \in M} u(x, m)-\inf _{x \in M} u(x, m-1)\right) .
\end{aligned}
$$

We define the oscillation $\theta(t)=\sup _{x \in M} u(x, t)-\inf _{x \in M} u(x, t)$. Adding the above inequalities gives

$$
\theta(m-1)+\theta\left(m-\frac{1}{2}\right) \leq C(\theta(m-1)-\theta(m))
$$

Rearranging and setting $\delta=\frac{C-1}{C}<1$ yields

$$
\theta(m) \leq \delta \theta(m-1)
$$

By induction,

$$
\theta(t) \leq C \mathrm{e}^{-\eta t}
$$

where $\eta=-\log \delta$. Note that if $u(x, m-1)$ is constant, this inequality is still true. 
Fix $(x, t)$ in $M \times[0, \infty)$. Since

$$
\int_{M} \frac{\partial \tilde{\varphi}}{\partial t} \omega^{n}=0
$$

there exists a point $y$ in $M$ such that $\frac{\partial \tilde{\varphi}}{\partial t}(y, t)=0$.

$$
\begin{aligned}
\left|\frac{\partial \tilde{\varphi}}{\partial t}(x, t)\right| & =\left|\frac{\partial \tilde{\varphi}}{\partial t}(x, t)-\frac{\partial \tilde{\varphi}}{\partial t}(y, t)\right| \\
& =\left|\frac{\partial \varphi}{\partial t}(x, t)-\frac{\partial \varphi}{\partial t}(y, t)\right| \\
& \leq C \mathrm{e}^{-\eta t} .
\end{aligned}
$$

Consider the quantity $Q_{2}=\tilde{\varphi}+\frac{C}{\eta} \mathrm{e}^{-\eta t}$. Then by construction,

$$
\frac{\partial Q_{2}}{\partial t} \leq 0
$$

Since $Q_{2}$ is bounded and monotonically decreasing, it tends to a limit as $t \rightarrow \infty$, call it $\tilde{\varphi}_{\infty}$. But

$$
\lim _{t \rightarrow \infty} \tilde{\varphi}=\lim _{t \rightarrow \infty} Q_{2}-\lim _{t \rightarrow \infty} \frac{C}{\eta} \mathrm{e}^{-\eta t}=\tilde{\varphi}_{\infty} .
$$

To show that the convergence of $\tilde{\varphi}$ to $\tilde{\varphi}_{\infty}$ is actually $C^{\infty}$, suppose not. Then there exists a time sequence $t_{m} \rightarrow \infty$ such that for some $\varepsilon>0$ and some integer $k$,

$$
\left\|\tilde{\varphi}\left(x, t_{m}\right)-\tilde{\varphi}_{\infty}\right\|_{C^{k}}>\varepsilon, \quad \forall m .
$$

However, since $\tilde{\varphi}$ is bounded in $C^{\infty}$ there exists a subsequence $t_{m_{j}} \rightarrow \infty$ such that $\tilde{\varphi}\left(x, t_{m_{j}}\right) \rightarrow \tilde{\varphi}_{\infty}^{\prime}$ as $j \rightarrow \infty$ for some smooth function $\tilde{\varphi}_{\infty}^{\prime}$. By (7.2), $\tilde{\varphi}_{\infty}^{\prime} \neq \tilde{\varphi}_{\infty}$. This is a contradiction, since $\tilde{\varphi} \rightarrow \tilde{\varphi}_{\infty}$ pointwise. Hence the convergence of $\tilde{\varphi}$ to $\tilde{\varphi}_{\infty}$ is $C^{\infty}$.

We observe that $\tilde{\varphi}$ solves the parabolic flow

$$
\frac{\partial \tilde{\varphi}}{\partial t}=\log \frac{\operatorname{det}\left(g_{i \bar{j}}+\partial_{i} \partial_{\bar{j}} \tilde{\varphi}\right)}{\operatorname{det} g_{i \bar{j}}}-F-\int_{M} \frac{\partial \varphi}{\partial t} \omega^{n} .
$$

Taking $t$ to infinity, we see that $\tilde{\varphi}_{\infty}$ solves the elliptic Monge-Ampère equation

$$
\log \frac{\operatorname{det}\left(g_{i \bar{j}}+\partial_{i} \partial_{\bar{j}} \tilde{\varphi}_{\infty}\right)}{\operatorname{det} g_{i \bar{j}}}=F+b,
$$


where

$$
b=\int_{M}\left(\log \frac{\operatorname{det}\left(g_{i \bar{j}}+\partial_{i} \partial_{\bar{j}} \tilde{\varphi}_{\infty}\right)}{\operatorname{det} g_{i \bar{j}}}-F\right) \omega^{n} .
$$

This combined with Theorem 5.1 completes the proof of the main theorem, and also provides a parabolic proof of the main theorem in [26].

\section{Acknowledgments}

The author would like to thank his thesis advisor Ben Weinkove for countless helpful discussions and advice. The author would also like to thank Valentino Tosatti for helpful suggestions. The author thanks the referee for a careful reading of the first version of this paper and for making a number of helpful suggestions and comments.

The contents of this paper will appear in the author's forthcoming $\mathrm{PhD}$ thesis.

\section{References}

[1] T. Aubin, Équations du type Monge-Ampère sur les variétés kählériennes compactes, Bull. Sci. Math. (2) 102(1) (1978), 63-95.

[2] E. Bedford and B.A. Taylor, Variational properties of the complex Monge-Ampère equation, II. Intrinsic norms, Amer. J. Math. 101 (1979), 1131-1166.

[3] E. Calabi, On Kähler manifolds with vanishing canonical class, Algebraic geometry and topology. A symposium in honor of S. Lefschetz, pp. 78-89, Princeton University Press, Princeton, N.J., 1957.

[4] H.-D. Cao, Deformation of Kähler metrics to Kähler-Einstein metrics on compact Kähler manifolds, Invent. Math. 81 (1985), 359-372.

[5] X.X. Chen, The space of Kähler metrics, J. Differential Geom. 56 (2000), 189-234.

[6] S.S. Chern, H.I. Levine and L. Nirenberg, Intrinsic norms on a complex manifold, 1969 Global Analysis (Papers in Honor of K. Kodaira) pp. 119-139, University Tokyo Press, Tokyo.

[7] Cherrier, P. Équations de Monge-Ampère sur les variétés Hermitiennes compactes, Bull. Sc. Math. (2) 111 (1987), 343-385. 
[8] S. Dinew and S. Kołodziej, Pluripotential estimates on compact Hermitian manifolds, preprint, arXiv::0910.3937.

[9] S.K. Donaldson, Symmetric spaces, Kähler geometry and Hamiltonian dynamics, Northern California Symplectic Geometry Seminar, 13-33, Amer. Math. Soc. Transl. Ser. 2, 196, Amer. Math. Soc., Providence, RI, 1999.

[10] L.C. Evans, Classical solutions of fully nonlinear, convex, second order elliptic equations, Comm. Pure Appl. Math. 25 (1982), 333-363.

[11] J. Fu and S.-T. Yau, The theory of superstring with flux on nonKähler manifolds and the complex Monge-Ampère equation, J. Differential Geom. 78(3) (2008), 369-428.

[12] B. Guan and Q. Li, Complex Monge-Ampère equations and totally real submanifolds, Adv. Math. 225(3) (2010), 1185-1223.

[13] D. Gilbarg and N.S. Trudinger, Elliptic partial differential equations of second order, Springer-Verlag, Berlin, Heidelberg, New York, 1983.

[14] B. Guan, The Dirichlet problem for the complex Monge-Ampère equation and regularity of the pluricomplex Green's function, Comm. Anal. Geom. 6(4) (1998), 687-703; correction in Comm. Anal. Geom. 8(1) (2000), 213-218.

[15] P.-F. Guan, Extremal functions related to intrinsic norms, Ann. Math. 156 (2002), 197-211.

[16] N.V. Krylov, Boundedly nonhomogeneous elliptic and parabolic equations, Izvestia Akad. Nauk. SSSR 46 (1982), 487-523, english translation in Math. USSR Izv. 20(3) (1983) 459-492.

[17] G. Lieberman, Second order parabolic differential equations, World Scientific, Singapore, New Jersey, London, Hong Kong, 1996.

[18] P. Li and S.-T. Yau, On the parabolic kernel of the Schrödinger operator, Acta Math. 156 (1986), 153-201.

[19] Mabuchi, T. Some symplectic geometry on compact Kähler manifolds. I, Osaka J. Math. 24 (1987), 227-252.

[20] D.H. Phong and J. Sturm, The Dirichlet problem for degenerate complex Monge-Ampère equations, Comm. Anal. Geom. 18(1) (2010), 145-170. 
[21] S. Semmes, Complex Monge-Ampère and symplectic manifolds, Amer. J. Math. 114 (1992), 495-550.

[22] Y.-T. Siu, Lectures on Hermitian-Einstein metrics for stable bundles and Kähler-Einstein metrics, DMV Seminar, 8, Birkhäuser Verlag, Basel, 1987.

[23] J. Streets and G. Tian, Regularity results for pluriclosed flow, preprint, arXiv:1008.2794.

[24] G. Székelyhidi and V. Tosatti, Regularity of weak solutions of a complex Monge-Ampère equation, preprint, arXiv:0912.1808.

[25] V. Tosatti and B. Weinkove, Estimates for the complex Monge-Ampère equation on Hermitian and balanced manifolds, Asian J. Math. 14(1) (2010), 19-40.

[26] V. Tosatti and B. Weinkove, The complex Monge-Ampère equation on compact Hermitian manifolds, J. Amer. Math. Soc. 23(4) (2010), 11871195.

[27] N.S. Trudinger, Fully nonlinear, uniformly elliptic equations under natural structure conditions, Trans. Amer. Math. Soc. 278(2) (1983), 751769 .

[28] B. Weinkove, The J-Flow, the Mabuchi energy, the Yang-Mills flow and Multiplier Ideal Sheaves, PhD thesis, Columbia University, 2004.

[29] S.-T. Yau, On the Ricci curvature of a compact Kähler manifold and the complex Monge-Ampère equation, I, Comm. Pure Appl. Math. 31(3) (1978), 339-411.

\section{Mathematics Department \\ University of CALIFornia \\ SAN DIEGO \\ 9500 Gilman Drive \#0112 \\ LA JOLLA \\ CA 92093 \\ USA \\ E-mail address: mfgill@math.ucsd.edu}

Received OCtoBer 26, 2010 
